\title{
Hemoglobin Barts
}

National Cancer Institute

\section{Source}

National Cancer Institute. Hemoglobin Barts. NCI Thesaurus. Code C103920.

A severe and rare form of alpha thalassemia characterized by the absence of alpha

globin chains. It results in hydrops fetalis, severe anemia, hepatosplenomegaly, heart

defects, and genitourinary abnormalities. It leads to death in utero or shortly thereafter. 